\title{
ÍNDICE GLICÉMICO E INSULINEMICO DE DOS TIPOS DE PASTA DE PRESENTACIÓN LARGA Y CORTA EN INDIVIDUOS SANOS
}

\section{GLYCEMIC AND INSULINEMIC INDEX OF TWO TYPES OF PASTA OF SHORT AND LONG PRESENTATION IN HEALTHY SUBJECTS}

\author{
Sandra Hirsch B., Gladys Barrera A., Laura Leiva B., \\ M. Pía de la Maza C., Daniel Bunout B.
}

\begin{abstract}
Laboratorio Envejecimiento y Enfermedades Crónicas Relacionadas con la Nutrición, Instituto de Nutrición y Tecnología de los Alimentos (INTA), Universidad de Chile. Santiago, Chile.
\end{abstract}

\begin{abstract}
AIM: To measure the glucose and insulin responses of a standard dry pasta and a dry pasta enriched with egg in their long and short presentation. MATERIAL AND METHODS: The glycemic index (GI) and the insulin index (II) were determined in a randomized, cross over protocol in 10 healthy volunteers between 26 and 58 years of age. Each subject underwent ten tests: six with the standard meal (bread) and four with the study pastas. Every study pasta was evaluated 10 times. Blood glucose and insulin levels were measured in the fasted state and over the 180 min following commencement of consumption of the foods. The Area Under the Curve (AUC) was calculated for the values above baseline for the 3-hour period following the meal. GI and II were calculated as the ratio of glucose and insulin AUCs for a given test meal and the AUCs for the standard meal. RESULTS: Both type of pasta in their long and short presentation had lower GI than the standard meal (long pasta A: 47.8 $54.4 \%$,pasta B: $58.6 \pm 95.4 \%$; short pasta A:71.4 \pm 32.8 , pasta B: $79.9 \pm 47.6 \% \%$ p<.001). The II was lower with the two type of large pasta compare to the standard meal and to the two type of short pasta $(p<.005)$. CONCLUSIONS: This study has shown that dry pasta with or without addition of egg put in the mass, are low GI food. Long dry pasta have lower insulin response.
\end{abstract}

Key words: large pasta, short pasta, glycemic index.

Este trabajo fue recibido el 25 de Mayo de 2010 y aceptado para ser publicado el 14 de Octubre de 2010.

\section{INTRODUCCIÓN}

El índice glicémico (IG) es una clasificación de los alimentos, basada en la respuesta postprandial de la glucosa sanguínea, comparada con un alimento de referencia (pan blanco o solución de glucosa). Se ha propuesto que el IG de los alimentos puede ayudar a mantener el control de peso de un individuo. También se ha demostrado en estudios a corto plazo que dietas de bajo IG y ricas en fibra pueden retardar la sensación de apetito y subsecuentemente disminuir el consumo energético al compararlas con dietas de alto IG (1).

Una dieta con un bajo IG $(\leq 70 \%)$, se considera saludable, particularmente en la prevención de obesidad, diabetes tipo 2 y enfermedades cardiovasculares. Más aún, en enfermos diabéticos permite un mejor control glicémico, disminuye la demanda de insulina y permite un mejor manejo de los lípidos sanguíneos $(2,3)$. El IG se asocia positivamente con el síndrome metabólico y la resistencia a insulina. En la cohorte de Framingham se demostró que aquellos individuos que consumían productos de alto IG tenían $41 \%$ de mayor riesgo de tener un síndrome metabólico, que aquellos que consumían dietas de bajo IG (4). En Chile, en los últimos 20 años la mayor incidencia de obesidad y síndrome metabólico, ha ido en paralelo con el aumento del consumo de alimentos procesados y de carbohidratos refinados (alto IG).

El IG de un alimento depende principalmente de la velocidad de digestión, es decir, mientras mayor es la velocidad de digestión de un determinado alimento mayor será el IG. En consecuencia la respuesta glicémica de un alimento puede variar por diferentes factores, el más importante es el tipo de carbohidrato, así por 
ejemplo, la glucosa tiene el IG promedio más alto $138 \%$, luego maltosa $105 \%$, sacorosa $75 \%$ y la fructuosa, el de menor IG (30\%). La velocidad de vaciamiento gástrico también influye en el IG, aquellas preparaciones que enlentecen el vaciamiento por su contenido en grasa y proteínas, disminuyen el IG. El contenido de agua, pH, tipo de fibra, tiempo de cocción y la temperatura que se ingiere un alimento, también modifican la respuesta glicémica (5).

Las pastas secas (industrializadas) culturalmente se han asociado con alimentos hiperglicemiantes, incluso más que el pan, arroz y papas. No obstante, las pastas se manufacturan a base de harina dura de trigo y otros cereales, llamado semolina, a diferencia del pan que se prepara con harina fina, lo que supone que la respuesta insúlinica y glicémica debiera ser menor que la del pan.

El objetivo de este estudio fue determinar el índice glicémico e insulinémico de una pasta seca corriente y de una enriquecida con huevo en su presentación larga y corta consumidas habitualmente por la población chilena.

\section{SUJETOS Y MÉTODOS}

El estudio se realizó en 2 etapas. En la primera se midió el IG e II de una pasta seca corriente (Lucchetti ${ }^{\mathrm{R}}$ ) y de una enriquecida con huevo $\left(\right.$ Talliani ${ }^{\mathrm{R}}$ ) (tabla 1) en su presentación larga (espagueti) y después de 6 meses se realizó la medición de IG e II del mismo tipo de pastas pero en presentación corta (corbata) en los mismos individuos para evitar factores confundentes.

Participaron 10 voluntarios sanos de ambos sexos ( 8 mujeres y 2 varones), con edades entre 26 y 58 años y con glicemia en ayunas $<99 \mathrm{mg} / \mathrm{dl}$ e índice de masa corporal $\leq 30 \mathrm{~kg} / \mathrm{m}^{2}$.

Los mismos sujetos aceptaron participar en ambas etapas, mediante la firma de un consentimiento informado por escrito en cada uno de los estudios. El proyecto fue aprobado por el Comité de Etica del Instituto de Nutrición y Tecnología de los Alimentos (INTA), Universidad de Chile.

Cada individuo seleccionado se sometió en forma aleatoria a 10 pruebas. En cada etapa se realizaron 3 pruebas para el alimento estándar y 2 para las pastas en estudio (una evaluación por pasta), con un intervalo de al menos una semana entre cada evaluación, de acuerdo a las recomendaciones de Wolever (6).

El alimento estándar fue pan blanco (110 g) «marraqueta» fresco obtenido de una panadería previamente estandarizada, con un aporte total de $55 \mathrm{~g}$ de hidratos de carbono. A todos los voluntarios se les realizó el primer estudio, en ambas etapas, con el alimento estándar.

El día del protocolo, los sujetos asistieron al INTA a las 08:30 h, con indicación previa de una dieta liviana y un ayuno de 10 horas, se instaló una vía venosa en el

\section{TABLA 1}

Composición de las pastas utilizadas para determinación del Indice Glicémico.

\begin{tabular}{lcccc} 
& $\begin{array}{c}\text { PASTA A } \\
\mathbf{1 0 0 g}\end{array}$ & $\begin{array}{c}\text { Larga y corta } \\
\text { Porción } \mathbf{8 0 ~ g}\end{array}$ & $\begin{array}{c}\text { PASTA B } \\
\mathbf{1 0 0} \mathbf{g}\end{array}$ & $\begin{array}{c}\text { Larga y corta } \\
\text { Porción } \mathbf{8 0} \text { g }\end{array}$ \\
\hline Agregado de huevo & - & - & + & + \\
Sémola de trigo duro & + & + & + & + \\
Energía( Kcal) & 338 & 270 & 359 & 287 \\
Proteínas (g) & 10 & 8 & 12 & 10 \\
Grasa (g) & 2 & 1.6 & 3 & 2 \\
Colesterol(mg) & 0 & 0 & 45 & 36 \\
Hidrato de Carbono (G) & 70 & 56 & 71 & 57 \\
Sodio (mg) & 20 & 16 & 21 & 17 \\
Tiamina (mg) & 0,9 & $51^{*}$ & 0,9 & $51^{*}$ \\
Riboflavina (mg) & 0,3 & $15^{*}$ & 0,3 & $15^{*}$ \\
Niacina (mg) & 5,7 & $25^{*}$ & 5,7 & $25^{*}$ \\
Hierro (mg) & 3 & $17^{*}$ & 3 & $17^{*}$ \\
\hline * \% recomendación diaria & & & & \\
\hline
\end{tabular}


antebrazo y se tomó una muestra inicial de sangre (3 ml) y 15 minutos, después se tomó una segunda muestra para obtener el valor de glicemia e insulinemia de la línea de base, que correspondió al tiempo 0. Inmediatamente de tomadas las muestras basales, cada sujeto consumió en un período de $10 \mathrm{~min}$, la pasta asignada aleatoriamente, o pan blanco, junto con $300 \mathrm{ml}$ de agua. Cada sujeto ingirió $85 \mathrm{~g}$ de pasta $\mathrm{A}$ o B en presentación larga o corta, que aporta $55 \mathrm{~g}$ de hidratos de carbono, preparada al dente (hervor de 8 minutos) y cada porción se le agregó $5 \mathrm{ml}$ (una cucharadita) de aceite de pepa de uva.

Posteriormente, se obtuvieron muestras de sangre (3 ml) a los $15,45,60,90,120,150$ y $180 \mathrm{~min}$, para la medición de glucosa e insulina. Durante el período de prueba, los sujetos estaban cómodamente sentados en una sala en un ambiente tranquilo.

Las muestras de sangre se centrifugaron a 3.000 rpm por $15 \mathrm{~min}$, a $4^{\circ} \mathrm{C}$. La glucosa fue medida por el método de glucosa-oxidasa (GOD PAP) y la insulina se midió por RIA utilizando kits comerciales (DPC, Los Ángeles, CA, EE.UU.).

\section{MÉTODOS ESTADÍSTICOS Y ANÁLISIS DE DATOS}

Con las concentraciones de glucosa e insulina del suero, se determinó el área bajo la curva $(\mathrm{ABC})$, calculada con los valores por encima de la línea de base en el período de $3 \mathrm{~h}$ después de ingerido el alimento. Los valores bajo la línea de base se obtuvieron con el cálculo de Wolever (6).
Los IG e II fueron calculados como la razón entre el $\mathrm{ABC}$ para un alimento de prueba dado y el promedio para la tres $\mathrm{ABC}$ del alimento estándar (considerado el $100 \%$ ) de un mismo sujeto, multiplicado por 100.

Los análisis estadísticos fueron realizados usando el Programa Stata 9.1 para Windows (Stata Corporation, Texas USA). La comparación entre las pastas y el alimento estándar se realizó mediante Kruskal-Wallis para series no paramétricas.

Se consideró la probabilidad $\mathrm{p}=0.05$ como significativa. El código de las pastas se abrió una vez realizado los análisis estadísticos.

La empresa que financió el estudio (TRESMONTES LUCCHETTI) no participó en el diseño ni análisis de los resultados, ni en la decisión de la publicación de los resultados.

\section{RESULTADOS}

Todos los sujetos completaron los 10 protocolos planificados durante los dos períodos. El ABC para glucosa e insulina de cada pasta se muestra en la tabla 2. Las $\mathrm{ABC}$ de la glucosa e insulina para la pasta A y B en la presentación larga fueron semejantes, pero menores que las del alimento estándar $(\mathrm{p}<0.02)$. En cambio las ABCs de las mismas pastas en presentación corta tendieron a ser menores, pero sin significación estadística.

El IG de ambas pastas largas fue significativamente menor que el del alimento estándar (pasta A: $47,8 \pm 54,4 \%$, pasta $\mathrm{B}: 58,6 \pm 95,4 \%) \mathrm{p}=0.006$, sin haber diferencias significativas entre ambas. La variabilidad

\section{TABLA 2}

\section{Area bajo la curva (ABC) de glucosa e insulina del producto estándar y de las 2 pastas en presentación larga y corta (promedio \pm desviación estándar).}

\begin{tabular}{|c|c|c|c|c|}
\hline & Alimento estándar & Pasta A & Pasta B & $\mathbf{p}$ \\
\hline \multicolumn{5}{|l|}{ PASTA LARGA } \\
\hline $\begin{array}{l}\text { ABC GLUCOSA } \\
\mathrm{mg} * 180 \mathrm{~min} / \mathrm{dl}\end{array}$ & $2953,2 \pm 1976,5$ & $1296,2 \pm 1164,5^{*}$ & $919,5 \pm 1053,6^{*}$ & 0,002 \\
\hline $\begin{array}{l}\text { ABC INSULINA } \\
\text { uIU*180 } \mathrm{min} / \mathrm{ml}\end{array}$ & $4019,5 \pm 1663$ & $1503,2 \pm 925.1$ & $1574,5 \pm 862,7$ & 0,001 \\
\hline \multicolumn{5}{|l|}{ PASTA CORTA } \\
\hline $\begin{array}{l}\text { ABC GLUCOSA } \\
\mathrm{mg} * 180 \mathrm{~min} / \mathrm{dl}\end{array}$ & $3428,3 \pm 1976,5$ & $2367,1 \pm 995,5$ & $2730,1 \pm 1507,8$ & ns \\
\hline $\begin{array}{l}\text { ABC INSULINA } \\
\text { uIU*180 } \mathrm{min} / \mathrm{ml}\end{array}$ & $4601.1 \pm 5120.5$ & $3088,8 \pm 2368,8$ & $4573,2 \pm 2489,2$ & ns \\
\hline
\end{tabular}


interindividual fue de $114 \%$ para la pasta A y $163 \%$ para la pasta B (tabla 3).

El IG de los dos tipos de pasta corta fue menor que el del alimento estándar (pasta A:71.4 \pm 32.8 , pasta B: $79,9 \pm 47,6 \% \%) \mathrm{p}=0.013$ con una variabilidad interindividual de $59,5 \%$ para la pasta A y $45,8 \%$ para la pasta B. Tampoco se observó diferencia entre las dos tipos de pasta corta. Al comparar IG de cada tipo pasta de acuerdo a la presentación no se observó diferencia entre las pastas larga y corta para el tipo $\mathrm{A}(\mathrm{p}=0.35)$, como tampoco para las pastas tipo $\mathrm{B}(\mathrm{p}=0.27)$ (tabla 3$)$.

El II de los dos tipos de pastas largas fue menor que el alimento estándar y ambas pastas de presentación corta $(\mathrm{p}<0.013)$. El II de los mismos tipos de pasta, pero en presentación corta, no se diferenció del alimento estándar, como tampoco entre ellas (tabla 3). La variabilidad interindividual para la pasta A fue de $72.8 \%$ y $76.3 \%$ para la pasta $\mathrm{B}$.

\section{DISCUSIÓN}

Este estudio demuestra que las pastas industrializadas independiente de su presentación corta o larga tienen un IG menor que el alimento estándar (pan blanco) en individuos sanos. Resultados semejantes con ravioles con salsa de tomate y queso ( $\mathrm{IG}=43 \%$ ), obtuvo Fajkusova al evaluar el IG de varias preparaciones de consumo habitual7. En diabéticos tipo 1, se ha observado bajo régimen de terapia insulínica intensiva, que las pastas provocan una menor respuesta glicémica que los fideos de arroz, hamburguesa tailandesa y sándwich (8).

Estos resultados se deben posiblemente a los ingredientes de las pastas. En la industria de pastas se utiliza grano duro de trigo para la preparación de la masa, que corresponde a un hidrato de carbono complejo a diferencia del pan que utiliza harina fina. Como se sabe los Hidratos de Carbono complejos provocan una respuesta glicémica e insulínica menor que los refinados, debido a que su absorción es más lenta. Este efecto sería beneficioso para individuos con resistencia a la insulina o diabéticos.

Por otra parte, los alimentos con bajo IG se asocian a una mayor sensación de saciedad, debido a que retardan el vaciamiento gástrico. En general los alimentos que inducen una baja respuesta glicémica son ricos en fibra, prolongando así la distención gástrica, causando probablemente un aumento de los péptidos asociados a la saciedad. Este efecto no lo medimos, debido a que el diseño para el objetivo principal, no lo permitía. Sin embargo, en un estudio realizado recientemente, al comparar el efecto del pan de grano entero con de grano refinado y pasta, se observó que las pastas tenían una menor respuesta glicémica que los panes y mayor efecto de saciedad que el pan de harina refinada (9). Otro estudio no demostró efecto de alimentos de bajo IG, incluido pastas en la saciedad e índice de masa corporal después de 12 semanas de intervención con dieta isocalórica, pero con diferentes IG, en mujeres con sobrepeso (10).

La ingesta de hidratos de carbono de alto IG se asocia a un mayor riesgo cardiovascular asociado con estrés oxidativo, aún en individuos sin intolerancia a la glucosa o síndrome metabólico. En experimentos en humanos con dietas con $50 \mathrm{~g}$ de hidratos de carbono como glucosa o pan con harina refinada y pasta, mostraron que las pastas tenían un efecto insignificante sobre la activación de NF- $\kappa \mathrm{B}$, (que es regulado por el estado oxidativo) a diferencia del pan y glucosa que lo activaban (11).

\section{TABLA 3}

\section{Indice glicémico e insulínico de las $\mathbf{2}$ pastas en presentación larga y corta (promedio \pm desviación estándar).}

\begin{tabular}{|c|c|c|c|c|}
\hline & Alimento estándar & Pasta A & Pasta B & $\mathbf{p}$ \\
\hline \multicolumn{5}{|c|}{ PASTA LARGA } \\
\hline $\mathrm{IG} \%$ & 100 & $47,8 \pm 54,4$ & $58,6 \pm 95,4$ & $0,006^{*}$ \\
\hline $\mathrm{II} \%$ & 100 & $39,2 \pm 22 ., 8 \alpha$ & $41,9 \pm 22,4 \alpha$ & $0,007 *$ \\
\hline \multicolumn{5}{|c|}{ PASTA CORTA } \\
\hline IG \% & 100 & $71,4 \pm 32,8$ & $79,9 \pm 47,6$ & $0,013^{*}$ \\
\hline II\% & 100 & $69,8 \pm 50,8$ & $117,6 \pm 89,7$ & ns \\
\hline $\begin{array}{l}\text { IG=índice glicém } \\
\text { Kruskal-Wallis: }\end{array}$ & $\begin{array}{l}\text { llínico } \\
\text { imento estándar. } \\
\text { pasta larga y corta } \mathrm{p}<0.05\end{array}$ & & & \\
\hline
\end{tabular}


Las pastas cortas y largas analizadas, tienen la misma formulación, sin embargo observamos una mayor respuesta insulínica con las patas corta y el IG aún cuando fue menor que el alimento estándar, tiende a ser mayor con las pastas cortas. Esta diferencia se puede deber a la superficie expuesta de la pasta que está determinada por la forma. Las pastas con más superficie expuesta como las corbatas (utilizada en este estudio), puede ser menos resistente a la cocción, pudiendo liberar más almidón al agua de cocción producto de la agitación al momento de cocinarla. Este almidón puede quedar adherido a la pasta una vez cocida, pudiendo causar una mayor respuesta insulínica.

Debemos reconocer que la debilidad de este estudio es la gran variabilidad interindividual. Tanto estudios previos realizados por nosotros (12), como otros estudios, han presentado esta gran variabilidad, independiente del alimento o fórmula utilizada. Probablemente esta variabilidad represente la realidad de la respuesta glicémica de los alimentos en un mismo individuo o interindividual, debido a factores inmanejables, tales como, condición de estrés de cada sujeto en el día del estudio (13) o el tipo de alimentos de la última comida antes de medir el IG de un alimento en estudio.

En conclusión los dos tipos de pasta en sus presentaciones largas y cortas tienen una menor respuesta glicémica que el pan blanco (marraqueta). Al parecer las pastas largas se asocian a un menor IG que las corta y una menor respuesta insulínica.

\section{RESUMEN}

Introducción: Las pastas secas (industrializadas) se caracterizan por ser manufacturadas a base de harina dura de trigo y otros cereales, llamado semolina, a diferencia del pan que se prepara con harina fina, lo que supone que la respuesta insulinémica y glicémica debiera ser menor que la del pan. Objetivo: Medir la respuesta glicémica e insulinsulinémica de una pasta seca estándar y una pasta seca enriquecida con huevo, de presentación larga (espagueti) y corta (corbata). Material y Métodos: El índice glicémico (IG) e índice insulinémico (II) se determinaron en 10 sujetos sanos entre 26 y 58 años de edad. En forma aleatoria a cada sujeto se le midió la glicemia e insulina en ayuno y después de ingerir en forma isoglucídica alimento estándar (pan marraqueta) o los 2 tipos de pasta en presentación larga o corta a los $15,45,60,90,120,150$ y 180 minutos. Posteriormente se calculó el área bajo la curva $(\mathrm{ABC})$ de glucosa e insulina en cada sujeto. Los IG e II fueron calculados como la razón entre el $\mathrm{ABC}$ para un alimento de prueba dado y el ABC del alimento estándar (considerado el 100\%). RESULTADOS: Ambos tipos de pasta en presentación larga y corta presentaron menor IG que el alimento estándar (marraqueta) ( pasta larga A: 47,8 $\pm 54,4$, pasta larga B: $58,6 \pm 95,4 \%$; pasta corta A:71, $4 \pm 32,8 \%$, pasta corta B: $79,9 \pm 4, .6 \%$ p<.001). El II de los dos tipos de pasta larga fue menor que el alimento estándar y los mismos tipos de pasta, pero cortas $(\mathrm{p}<.005)$. El II de las pastas cortas no se diferenció del alimento estándar. Conclusión: Este estudio demostró que las pastas secas con o sin adición de huevo tienen una menor respuesta glicémica que el alimento estándar, por lo tanto, se pueden clasificar como alimento de bajo IG. Además las pastas de presentación larga tienen una menor respuesta insulinémica.

Palabras clave: pasta larga, pasta corta, índice glicémico

Dirigir la correspondencia a:

Dra.

Sandra Hirsch Birn

Laboratorio Envejecimiento y Enfermedades

Crónicas Relacionadas con la Nutrición

Instituto de Nutrición y Tecnología de los Alimentos (INTA)

Universidad de Chile

Santiago, Chile

Teléfono: 9781495

Fax: 2214030

E-mail: shirsch@inta.cl

Agradecimientos: Financiamiento TRESMONTES LUCCHETTI.

\section{BIBLIOGRAFÍA}

1. Wolever TM, Jenkins DJ. The use of the glycemic index in predicting the blood glucose response to mixed meals. Am J Clin Nutr 1986; 43: 167-72.

2. Jenkins $\mathrm{Dj}$, Kendall Cw, Augustin Ls, Franceschi S, Hamidi M, Marchie A Et Al. Glycemic index: overview of implications in health and disease. Am J Clin Nutr 2002; 76: 266S-73S. Review.

3. Pi Sunyer F. Glycemic index and disease. Am J Clin Nutr 2002;76: 290S-98S.

4. McKeown NM, Meigs JB, Liu S, Saltzman E, Wilson PW, Jacques PF. Carbohydrate nutrition, insulin resistance, and the prevalence of the metabolic syndrome in the Framingham Offspring Cohort. Diabetes Care. 2004;27:538-46.

5. Radulian G, Rusu E, Dragomir A, Posea M. Metabolic effects of low glycaemic index diets. Nutr J. 2009; 29;8:5.

6. Wolever TM, Jenkins DJ, Jenkins AL, Josse RG. The glycemic index: methodology and clinical implications. Am J Clin Nutr 1991; 54: 846-54. 
7. Fajkusova Z, Jadviscokova T, Pallayova M, Matuskova V, Luza J, Kuzmina G. Glycaemic index of selected foodstuffs in healthy persons.Biomed Pap Med Fac Univ Palacky Olomouc Czed Repub 2007;151.257-61.

8. Macdonald K, Lowe Jm, Barker D, Mensch M, Attia J. Effect of popular takeaway foods on blood glucose levels in type 1 diabetes mellitus patients on intensive insulin therapy. Int J Clin Pract. 2009;63:189-94.

9. Kristensen M, Jensen MG, Riboldi G, Petronio M, Bügel S, Toubro S, Tetens I, Astrup A. Wholegrain vs. refined wheat bread and pasta. Effect on postprandial glycemia, appetite, and subsequent ad libitum energy intake in young healthy adults. Appetite. 2010;54:163-9.

10. Aston LM, Stokes CS, Jebb SA. No effect of a diet with a reduced glycaemic index on satiety, energy intake and body weight in overweight and obese women. Int J Obes (Lond). 2008; 32(1):160-5.

11. Dickinson S, Hancock DP, Petocz P, Ceriello A, Brand-Miller J. High-glycemic index carbohydrate increases nuclear factor-kappaB activation in mononuclear cells of young, lean healthy subjects. Am J Clin Nutr. 2008;87:1188-93.

12. Gattás V, Barrera G, Leiva L, De La Maza MP, Bunout D, Steenhout P, Klassen P, Voss T, Hirsch S. Glycemic and insulin indices of tube feeding formulas in healthy adults. Rev Med Chile. 2007;135:879-84.

13. Derosa G, Salvadeo SA, Mereu R, D'Angelo A, Ciccarelli L, Piccinni MN, Ferrari I, Gravina A, Maffioli P, Tinelli C. Continuous glucose monitoring system in free-living healthy subjects: results from a pilot study. Diabetes Technol Ther. 2009; 11:159-69. 